\title{
Metastatic Lobular Carcinoma of the Breast Presenting with Small Bowel Metastases: Case Report and Literature Review
}

\author{
Rodrigo Arrangoiz*, María Cristina Ornelas, Janet Pineda-Díaz, Fernando Cordera, David Caba, \\ Eduardo Moreno, Enrique Luque-De-Leon, Manuel Muñoz
}

Sociedad Quirúrgica S. C. at the American British Cowdray Medical Center, Mexico City, Mexico

Email: *rodrigo.arrangoiz@gmail.com

How to cite this paper: Arrangoiz, R., Ornelas, M.C., Pineda-Díaz, J., Cordera, F., Caba, D., Moreno, E., Luque-De-Leon, E. and Muñoz, M. (2020) Metastatic Lobular Carcinoma of the Breast Presenting with Small Bowel Metastases: Case Report and Literature Review. Advances in Breast Cancer Research, 9, 1-11.

https://doi.org/10.4236/abcr.2020.91001

Received: November 21, 2019

Accepted: January 12, 2020

Published: January 15, 2020

Copyright $\odot 2020$ by author(s) and Scientific Research Publishing Inc. This work is licensed under the Creative Commons Attribution International License (CC BY 4.0).

http://creativecommons.org/licenses/by/4.0/

\begin{abstract}
Introduction: Invasive lobular carcinoma (ILC) is the second most common histologic type of breast cancer, representing $5 \%$ to $15 \%$ of invasive tumors. ILC tends to spread to bones, lungs, central nervous system, reproductive organs, and the gastrointestinal tract (GI tract). The most commonly affected organs in the GI tract are the stomach, small intestine, followed by colon and rectum. Case presentation: A 78-year-old woman who was referred to our institution after having a bowel obstruction that required a diagnostic laparoscopy where they identified an obstructing ulcerative lesion in the distal ileum that was managed with a segmental bowel resection. Pathology report showed an invasive lobular breast carcinoma that occluded $90 \%$ of the bowel lumen. A PET/CT scan revealed a left breast tumor with increased metabolism. The patient was staged as a clinical cT4b, cN0, cM1 left breast invasive lobular carcinoma (ER/PgR positive, HER-2 negative). She was managed with endocrine therapy with Letrozole (an eight-week course). A follow-up PET/CT showed a peritoneal hypermetabolic nodule adjacent to the previous ileal anastomosis. The lesion decreased in size and metabolic activity. In a multidisciplinary fashion, the endocrine therapy was extended for another three months. Another follow-up PET/CT scan was performed three months after the identification of the peritoneal implant that showed that the nodule increased in size and in metabolism. The lesion continued to decrease significantly in size and became metabolically inactivity. Due to the good breast response and the possibility that the ileal nodule could be a granuloma, she underwent an exploratory laparoscopy with excision of the peritoneal nodule, and a modified left radical mastectomy with immediate breast reconstruction (complex wound closure). The final pathology report of the nodule was negative for malignancy. She continued on endocrine therapy and underwent
\end{abstract}


whole breast irradiation four weeks after the operation. Currently, she is free of disease with no evidence of local, regional, or distant recurrence, and she is still on endocrine therapy. Discussion: The time interval between primary breast cancer and gastrointestinal involvement may range from synchronous presentation to as long as 30 years. The clinical manifestations in GI lobular breast cancer metastasis may range from non-specific complaints to acute GI symptoms, such as a bowel obstruction. There are multiple controversies in the management of ILC. Systemic treatment should be initiated as soon as possible. Indications for postmastectomy radiotherapy are also controversial, given the propensity for multifocal/multicentric tumors and late recurrences, sometimes in atypical locations. Five years of postoperative adjuvant hormonal therapy is an option for women with poor prognosis. Remissions are observed in $32 \%$ to $53 \%$ of patients. Conclusion: Metastatic lobular carcinoma of the breast has a wide range of clinical presentations. Patients with a history of breast cancer who present with new GI tumors should have these lesions evaluated for evidence of metastasis through histopathologic and immunohistochemical analysis, this will allow for appropriate management. Currently, breast cancer management involves a multidisciplinary approach including surgery, radiotherapy, and systemic medical therapy, and the treatment must be tailored to the patient's needs.

\section{Keywords}

Invasive Lobular Carcinoma of the Breast, Metastatic Lobular Carcinoma of the Breast, Metastatic Disease to the Small Bowel from Breast Cancer

\section{Introduction}

Worldwide, breast cancer is the most frequent malignancy and the most common cause of cancer related death in women aged 20 to 59 years [1]. Invasive lobular carcinoma (ILC) is the second most common histologic subtype of breast cancer, representing $5 \%$ to $15 \%$ of all invasive breast tumors [2] [3]. The reported incidence of ILC has ranged between $1 \%$ and 20\% [2]. The mean age at diagnosis of ILC is 57 years [4]. ILC has an increased propensity for multifocal and multicentric presentation [5]. Some of the known risk factors for ILC, that do not vary from other types of breast cancer, are hormone dependent and include age at menarche, age of menopause, age at first birth, nulliparity, obesity, and hormone therapy [3]. ILC is a diagnostic challenge given its infiltrative and insidious growth pattern, some women present with a defined palpable mass, while others display only vague skin thickening, diffuse nodularity, or may be clinically occult [3]. There are clear associations between histologic grade and other clinicopathological variables that portend a poor prognosis such as large size, positive lymph nodes, vascular invasion, estrogen receptors and androgen receptor negativity, and p53 positivity [6]. The work-up for ILC is very similar to other types of breast cancer and includes directed breast imaging like mammo- 
graphy, ultrasonography, and magnetic resonance imaging (when indicated) [1]. The pathologically disorganized pattern of ILC, frequently manifests as a scattered radiologic appearance and may result in misestimation of the extend of disease by imaging [1].

In lobular neoplasia (LN: atypical lobular hyperplasia and lobular carcinoma in situ) the risk of developing an invasive cancer is close to $0.5 \%$ per year, and when it is associated with a first-degree relative, the risk increases to $1 \%$ per year [1]. ILC tends to spread to bones, lungs, the central nervous system, reproductive organs, peritoneum, retroperitoneum, and the gastrointestinal tract (GI) [7] [8] [9]. The incidence of extrahepatic GI tract metastases observed in autopsy studies varies in the literature from $6 \%$ to $18 \%$ with the most commonly affected organs being the stomach, small intestine, followed by colon and rectum [10]. In ILC, a high rate of metastatic spread with an uncommon pattern of secondary deposits [11] [12] [13] [14] and high incidence of contralateral disease have been reported [3]. Commonly, the absence of specific symptoms related to the GI metastases from an ILC leads to a misdiagnosis of this entity [10].

Breast cancer treatment involves a multidisciplinary approach including surgery, radiotherapy, and systemic therapy. The surgical management of breast cancer is determined by the TNM staging system at presentation. Another fundamental element of treatment is appropriate evaluation and management of the axilla. Patients with early-stage breast cancer are candidates for surgery, either breast-conservative surgery (BCS) or mastectomy. Multiple randomized trials with long-term follow-up have demonstrated similar rates of locoregional control and survival with conservative therapy vs. mastectomy [15]. These trials included patients with ILC. Several studies have shown a survival advantage when the primary tumor is resected in patients with stage IV breast cancer, but this is controversial [1].

Most patients with locally advanced cancers (cT3, cT4/N1) should receive neoadjuvant systemic therapy. Patients with ILC are less likely to experience a complete pathologic response (CPR) to neoadjuvant therapy, with $\mathrm{CPR}$ ranging from $0 \%$ to $11 \%$ [16] [17]. Tumor biology is the primary determinant of response to neoadjuvant therapy, and the high ER positivity and low proliferative rates (low Ki-67) in ILC predisposes it to a leaser response [8]. Considering the known ER-rich nature of almost all ILCs, the use of neoadjuvant endocrine therapy is a good alternative. Favorable results from retrospective studies first led to an emerging interest in this approach [18] [19] [20]. Contemporary systemic therapies are largely influenced by tumor biology rather than histology. Hormone receptor-positive cancers should receive endocrine therapy. Chemotherapy is typically offered for locally advanced cancers and for early-stage cancers with high-risk features such as high-grade, large size, involved lymph nodes, and tumor with a molecular profile indicating high risk of recurrence.

Adjuvant breast radiotherapy (RT) reduces the risk of locoregional recurrence and death from breast cancer after surgery [1]. Additional regional nodal irradiation may also be indicated for those with involved lymph nodes or high-risk 
tumors [15]. There are no unique specifications for surveillance of ILC. For all treated non-metastatic breast cancers, the NCCN guidelines recommend a history and physical examination one to four times per year as clinically appropriate for five-years and then annually [21]. Adherence to endocrine therapy should be encouraged for those who will benefit from this type of therapy and yearly gynecologic assessment arranged for those without a previous hysterectomy.

We present a case of a 78-year-old woman who was referred to our institution after a small bowel obstruction that on a further evaluation was diagnosed as metastatic ILC of the breast.

\section{Case Presentation}

This is a 78-year-old woman, with a family history of breast cancer (mother had a unilateral invasive ductal carcinoma of the breast diagnosed at age), that 15 -years ago on a routine mammogram was diagnosed with a highly suspicious left breast tumor (Bi-RADS 5). At that moment, she decided not to treat it and used alternative medicine instead. She was under topical hormone replacement therapy for vulvar vaginal atrophy. Approximately six-month prior to her consultation she presented with a history of anorexia, weight loss, oral intolerance. She decided to go to a gastroenterologist for further work-up. An upper endoscopy was performed, demonstrating only severe gastritis and Helicobacter pylori infection, a colonoscopy was performed and was normal.

In a routine medical check-up, during the physical examination she was found to have asymmetry of the breasts, with a palpable mass in the left outer quadrant of the breast, that measured around eight centimeters in largest diameter, with skin retraction and apparent infiltration of pectoralis mayor muscle, with no palpable axillary lymphadenopathy (Figure 1). A CT scan of chest, abdomen and pelvis was performed and interpreted as negative for metastasis. A needle core biopsy was reported positive for ILC with a histologic grade 2, negative for lymphovascular invasion, with ER positive 79\%, PgR positive $66 \%$, negative for HER2, and low Ki-67 of $8 \%$. Subsequently, a PET/CT scan (Figure 2) revealed a hypermetabolic left breast tumor, and incidentally identified multiple dilated loops of small bowel filled with fluid with a transition point at the right mid abdomen and no inflammatory changes, negative for distal metastasis.

Two weeks after the study, she started to complain of abdominal pain, and signs and symptoms compatible with a bowel obstruction. She then presented to a local emergency department for persistent abdominal pain, where a diagnostic laparoscopy was performed, finding an obstructing ulcerated distal ileum tumor measuring $3 \mathrm{~cm} \times 1 \mathrm{~cm}$. A small bowel resection was performed. The pathology report showed an ILC that occluded $90 \%$ of the bowel lumen, positive for ER/PgR receptors, HER-2 negative. She was diagnosed as having a metastatic ILC, stage IV pT4b, cN1, cM1 of the left breast (ER/PgR positive, HER-2 negative). 


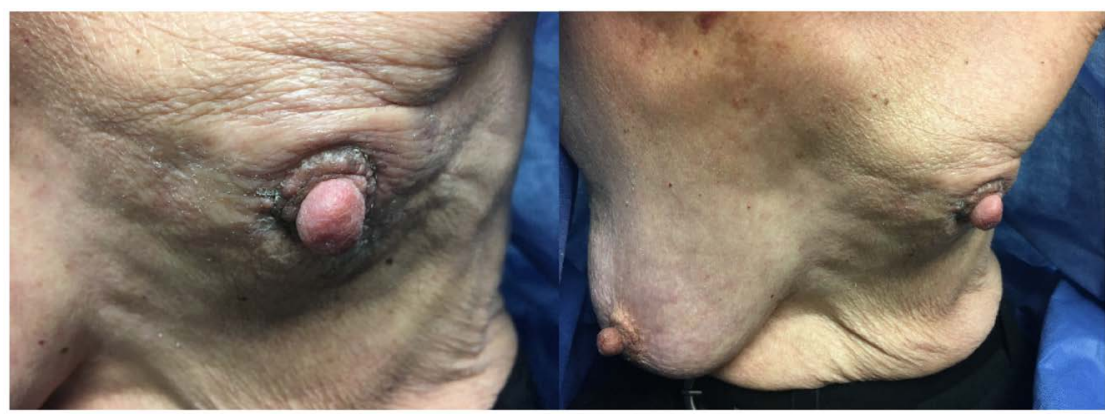

Figure 1. Left breast asymmetry with retraction of the skin and areola.

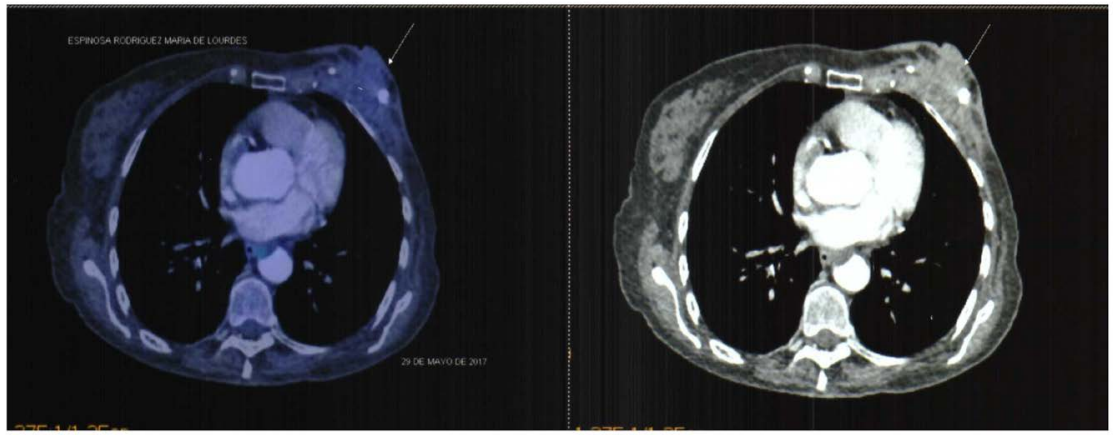

Figure 2. CT scan of the chest showing the left breast mass.

She was referred to our clinic and managed in a multidisciplinary fashion. She was started on endocrine therapy with Letrozole for eight-weeks. A follow-up PET/CT scan was performed after she completed the eight-weeks of therapy (Figure 3). The breast lesion had decreased in size and in metabolic activity. An isolated peritoneal hypermetabolic nodule was identified adjacent to the previous small bowel anastomosis. The endocrine therapy was extended for another 12 weeks. A follow-up PET/CT scan was performed after finishing the treatment, the peritoneal implant was still present, and hypermetabolic (Figure 4). The breast lesion had continued to decrease significantly in size and was hypermetabolic (SUV less than 2.5). It was decided in a multidisciplinary fashion to take the patient to the operating room and perform an exploratory laparoscopy with peritoneal nodule resection and a modified radical mastectomy with immediate bilateral breast reconstruction (complex wound closure) (Figure 5). She had an adequate postoperative course and she was discharged from the hospital on her third postoperative day.

She had an adequate recovery and continued endocrine therapy with Letrozole. She underwent radiation therapy receiving 30 sessions of $2 \mathrm{~Gy} / \mathrm{fr}$ until reaching a total of $60 \mathrm{~Gy}$ with good tolerance. On follow-up (more than two years) she has no evidence of disease. She is still on endocrine therapy with bi-annual clinic visits.

\section{Pathology}

The left breast and axillary lymph nodes demonstrated metastatic multicentric 


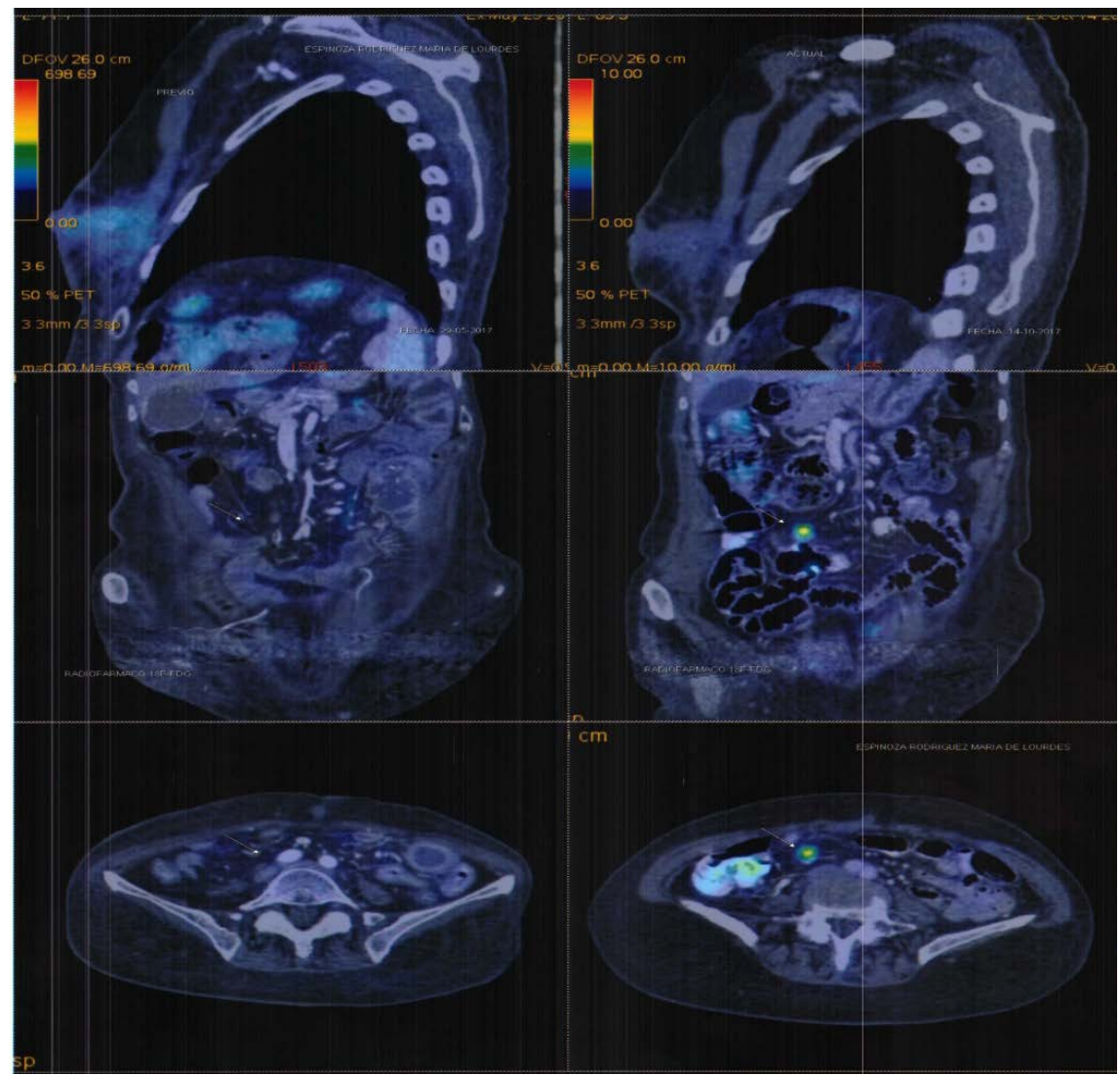

Figure 3. PET/CT scan that shows the hypermetabolic left breast mass and a peritoneal hypermetabolic nodule adjacent to the previous ileal anastomosis.

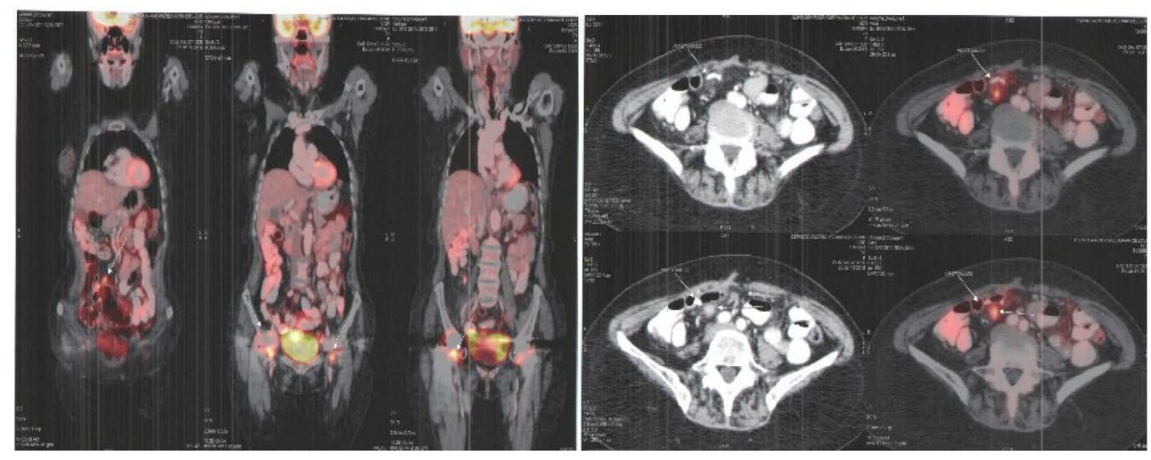

Figure 4. PET/CT scan with the peritoneal implant that increased in size and metabolism during follow-up.

ILC (in all the four quadrants), with two lymph nodes out of 17, positive for ILC. Immunochemistry performed with good positive and negative controls revealed tumoral cells positive for ER (99\%) and PgR (15\%). Her-2 was negative and Ki-67 positive in 2\% of the cells (Figure 6 and Figure 7). The peritoneal nodule biopsy showed an inflammatory granuloma negative for malignancy, just showing abundant lymphocytes mixed with some neutrophils in a fibrous stroma with giant multinucleated cells, constituting a foreign body xanthogranulomatous reaction (Figure 8). 


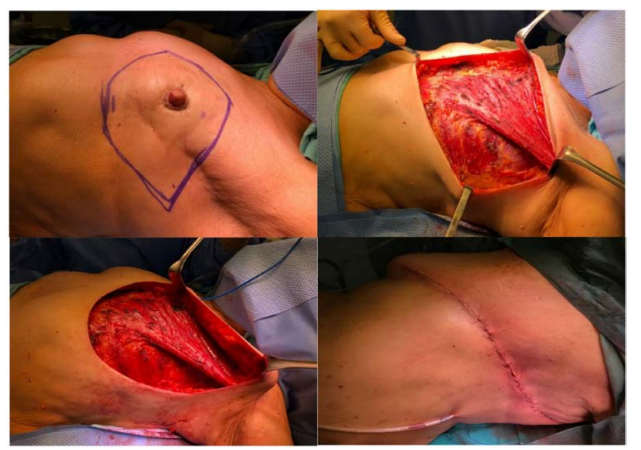

Figure 5. Intraoperative images of the left modified radical mastectomy.

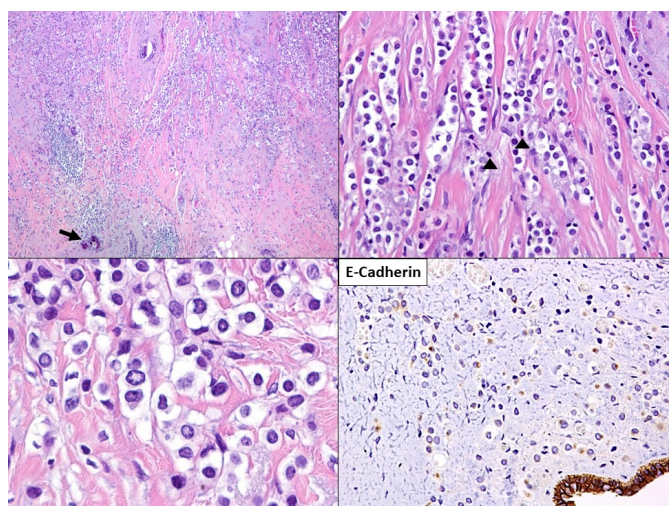

Figure 6. Product of left mastectomy. A. Panoramic microphotography that shows an infiltrating carcinoma on a fibrous and highly desmoplastic stroma with calcifications (arrow) and a dense lymphoid infiltrate ( $\mathrm{H} \& \mathrm{E} 10 \times)$. B. The neoplastic cells are arranged in single files that are loosely dispersed throughout a fibrous stroma, that do not form tubules, with scarce mitosis (arrow heads), (H \& E 40×). C. High power field that shows moderate nuclear pleomorphism and dispersed chromatin with some small evident nucleoli (H \& E 100×). D. Immunohistochemistry reaction for E-cadherin showing no membrane stain on the neoplastic cells. Internal control can be seen on the left corner.

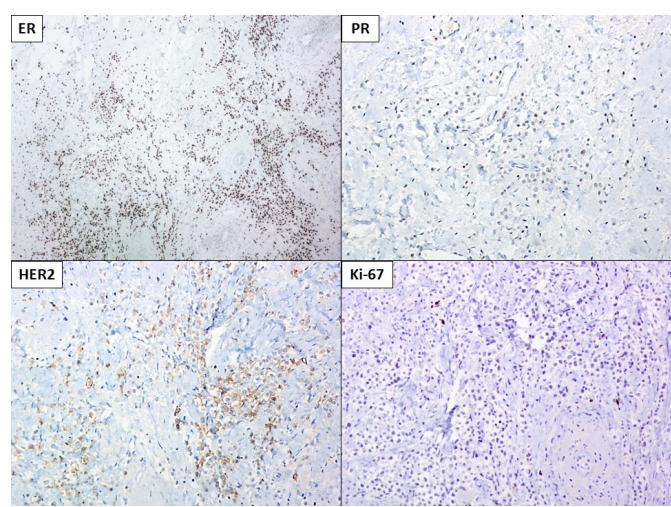

Figure 7. Immunohistochemistry prognostic panel of product of left mastectomy. A. Estrogen receptor immunohistochemistry reaction showing $99 \%$ of intense staining on the neoplastic cells. B. Progesterone receptor immunohistochemistry reaction showing 15\% of weak staining on the neoplastic cells. C. HER2 protein immunohistochemistry reaction showing complete weak membrane staining on more than $10 \%$ of the neoplastic cells, which defines a $2+$ or equivocal result. D. Ki-67 immunohistochemistry reaction showing a $2 \%$ of proliferation index. 


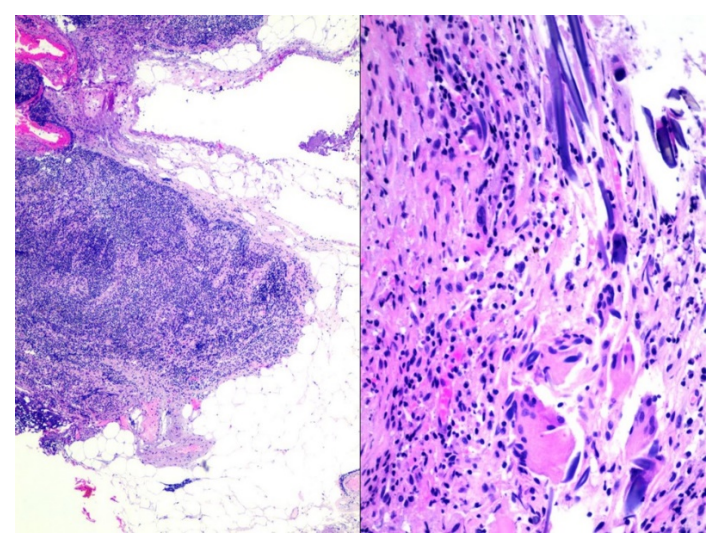

Figure 8. Referred as probable implant of breast carcinoma. A. Panoramic microphotography that shows fibroconnective and adipose tissue with inflammatory infiltrate $(\mathrm{H} \& \mathrm{E}$ $10 \times)$. B. High power microphotography that shows abundant lymphocytes mixed with some neutrophils in a fibrous stroma with giant multinucleated cells, constituting a foreign body xantogranulomatous reaction. Some strands of suture are visible on the right corner of the picture (H \& E 40x).

\section{Discussion}

Breast cancer is the most frequently diagnosed malignancy in women, and it is the most common cause of cancer death in women worldwide [1]. It accounts for $26 \%$ of all newly diagnosed cancers in females and is responsible for $15 \%$ of the cancer-related deaths in women [1]. It is also one of the most common malignancies that metastasize to the GI tract, commonly metastasizing to the stomach or small bowel [7] [8] [9]. The time interval between primary breast cancer and GI involvement may range from synchronous presentation to as long as 30 years after adequate management, but the reverse is also true, with the GI manifestations preceding the diagnosis of the breast cancer [14]. There tends to be a histological preference for the site for distant metastasis, invasive ductal carcinoma of the breast tends to metastasize more commonly to the liver, lung, and brain compared to lobular carcinoma that tends to spread to bones, gynecological organs, peritoneum, retroperitoneum, and gastrointestinal [7] [8] [9]. The reasons for this have not been identified. The clinical manifestations in GI ILC metastasis are variable. Symptoms may range from non-specific complaints to acute GI symptoms, such as a bowel obstruction.

The clinical manifestations that metastatic breast cancer to the GI tract can have are variable. Symptoms may range from non-specific complaints to acute GI symptoms. The diagnosis of metastatic lesions to the GI tract becomes even more unlikely on the rare occasion when it represents the first manifestation of breast cancer [7] [8] [9]. In our case the patient presented initially with GI symptoms that made her seek medical attention without taking into account the breast lesion.

Pathologic diagnosis of metastatic lesions can be difficult. Markers that help confirm the diagnosis are gross cystic disease fluid protein-15 (GCDFP-15), ER and PgR receptors. The GCDFP-15 and the ER and/or PR are positive in metas- 
tatic lesions of the breast, in contrast to most GI malignancies, which are usually negative. The signet-ring morphology of ILC may mimic gastric carcinoma. Some authors suggest that given the high incidence of breast cancer and the low incidence of diffuse-type gastric cancer in the USA, we should consider the presence of metastatic lobular carcinoma of the breast in every woman diagnosed with diffuse-type gastric cancer [18] [19] [20].

Multiple controversies exist in respect to the management of ILC. Systemic treatment should be initiated as soon as possible, preferably within six-weeks after surgery [1]. Treatment with chemotherapy, endocrine therapy or a combination of both is usually started in patients with ILC that metastasize to the GI tract [18]. Simultaneous use of radiotherapy and chemotherapy is not recommended since toxicity increases. Indications for postmastectomy radiotherapy, after having treated the primary ILC is also controversial, given the propensity for multifocal/multicentric tumors and late recurrences, sometimes in atypical locations [22]. Five-years of postoperative adjuvant hormonal therapy is an option for women with poor prognosis, especially in patients with positive lymph node disease [1]. Survival after diagnosis of GI metastases from a breast primary is poor with few patients surviving beyond two years, although survival up to nine-years has been reported [23]. Remissions are observed in $32 \%$ to $53 \%$ of patients [22].

\section{Conclusion}

Metastatic ILC of the breast has a wide range of clinical presentations. Small bowel metastasis from ILC is rare. Metastases from ILC to the GI tract must be excluded in a patient with a suspicious tumor of the breast, even though there is no confirmation of malignancy. Patients with a history of breast cancer who present with new gastrointestinal lesions should have these lesions evaluated for evidence of metastatic disease through histopathologic and immunohistochemical analysis, this will allow appropriate management. Nowadays, breast cancer treatment involves a multidisciplinary approach including surgery, radiotherapy, and systemic therapy. The treatment must be tailored to meet each individual's needs.

\section{Ethical Approval}

Written informed consent was obtained from the patient for publication of this case report and accompanying images. A copy of the written consent is available for review by the Editor-in-Chief of this journal.

\section{Author's Contributions}

Rodrigo Arrangoiz MS, MD, FACS was involved in the original idea for the project, drafting the manuscript and revising it critically for important intellectual content and had given final approval of the version to be published.

María Cristina Ornelas MD was involved in drafting the manuscript, revising it critically for important intellectual content, and organizing the team for criti- 
cal revision and had given final approval of the version to be published.

Janet Pineda-Díaz MD was involved in drafting the manuscript, revising it critically for important intellectual content, and obtaining the pathology images and had given final approval of the version to be published.

Fernando Cordera MD, FACS, David Caba MS, MD, MPH, Eduardo Moreno MD, Enrique Luque-de-Leon MD, Manuel Muñoz MD, FACS were involved in revising the manuscript critically for important intellectual content. They have given final approval of the version to be published.

\section{Conflicts of Interest}

The authors declare that they have no conflict of interest relating to the publication of this manuscript.

\section{References}

[1] Cárdenas, J., Bargalló, J.E., Maffuz, A., et al. (2017) Mexican Consensus of Diagnosis and Treatment of Breast Cancer. Seventh Revision Colima, Sociedad Mexicana de Oncología, 6-39.

[2] Mamtani, A. and King, T.A. (2018) Lobular Breast Cancer: Literature Review. Complex General Surgical Oncology, 1, 1-26.

[3] Sastre-Garau, X., Jouve, M., Asselain, B., Vincent-Salomon, A., Beuzeboc, P. and Dorval, T. (1996) Infiltrating Lobular Carcinoma of the Breast: Clinicopathological Analysis of 975 Cases with Reference to Data on Conservative Therapy and Metastatic Patterns. Cancer, 77, 113-120.

https://doi.org/10.1002/(SICI)1097-0142(19960101)77:1<113::AID-CNCR19>3.0.C $\underline{0 ; 2-8}$

[4] Sledge, G.W., Chagpar, A. and Perou, C. (2016) Collective Wisdom: Lobular Carcinoma of the Breast. American Society of Clinical Oncology Educational Book, 35, 18-21.

[5] Rakha, E.A. and Ellis, I.O. (2010) Lobular Breast Carcinoma and Its Variants. Seminars in Diagnostic Pathology, 27, 49-61.

https://doi.org/10.1053/j.semdp.2009.12.009

[6] Rakha, E.A., El-Sayed, M.E., Menon, S., et al. (2008) Histologic Grading Is an Independent Prognostic Factor in Invasive Lobular Carcinoma of the Breast. Breast Cancer Research and Treatment, 111, 121-127.

https://doi.org/10.1007/s10549-007-9768-4

[7] Schwarz, R.E., Klimsatra, D.S. and Turnbull, A.D. (1998) Metastatic Breast Cancer Masquerading as Gastrointestinal Primary. American Journal of Gastroenterology, 93, 111-114. https://doi.org/10.1111/j.1572-0241.1998.111 c.x

[8] Santini, D., Altomare, A., Vincenzi, B., Perrone, G., Bianchi, A. and Rabitti, C. (2006) An Increase of CA 19.9 as the First Clinical Sign of Ileocecal Valve Metastasis from Breast Cancer. In Vivo, 20, 165-168.

[9] Aurello, P., D’Angelo, F., Cosenza, G., Petrocca, S., Stoppacciaro, A. and Ramacciato, G. (2006) Gastric Metastasis 14 Years after Mastectomy for Breast Lobular Carcinoma: A Case Report and Literature Review. The American Journal of Surgery, 72, 456-460.

[10] Bamias, A., Baltayiannis, G., Kamina, S., et al. (2001) Rectal Metastases from Lobular Carcinoma of the Breast: Report of a Case and Literature Review. Annals of On- 
cology, 12, 715-718. https://doi.org/10.1023/A:1011192827710

[11] Pera, M., Riera, E., Lopez, R., et al. (2001) Metastatic Carcinoma of the Breast Resembling Early Gastric Carcinoma. Mayo Clinic Proceedings, 76, 205-207. https://doi.org/10.1016/S0025-6196(11)63129-7

[12] Lopez, M., Flores, L., Arias-Camisón, I., et al. (2010) Rectal Metástasis from Lobular Breast Carcinoma 15 Years after Primary Diagnosis. Clinical and Translational Oncology, 12, 150-153. https://doi.org/10.1007/S12094-010-0481-0

[13] Cervi, G., Vettoretto, N., Vinco, A., et al. (2001) Rectal Localization of Metastatic Lobular Breast Cancer: Report of a Case. Diseases of the Colon \& Rectum, 44, 453-455. https://doi.org/10.1007/BF02234749

[14] Arrangoiz, R., Papavasilious, P., Dushkin, H. and Frama, J.M. (2011) Case Report and Literature Review: Metastatic Lobular Carcinoma of the Breast an Unusual Presentation. International Journal of Surgery Case Reports, 2, 301-305. https://doi.org/10.1016/j.ijscr.2011.06.010

[15] Darby, S., McGale, P., Correa, C., et al. (2011) Effect of Radiotherapy after Breast-Conserving Surgery on 10-Year Recurrence and 15-Year Breast Cancer Death: Meta-Analysis of Individual Patient Data for 10,801 Women in 17 Randomised Trials. The Lancet, 378, 1707-1716. https://doi.org/10.1016/S0140-6736(11)61629-2

[16] Cocquyt, V.F., Blondeel, P.N., Depypere, H.T., et al. (2003) Different Responses to Preoperative Chemotherapy for Invasive Lobular and Invasive Ductal Breast Carcinoma. European Journal of Surgical Oncology, 29, 361-367.

https://doi.org/10.1053/ejso.2002.1404

[17] Tubiana-Hulin, M., Stevens, D., Lasry, S., et al. (2006) Response to Neoadjuvant Chemotherapy in Lobular and Ductal Breast Carcinomas: A Retrospective Study on 860 Patients from One Institution. Annals of Oncology, 17, 1228-1233. https://doi.org/10.1093/annonc/mdl114

[18] Lips, E.H., Mukhtar, R.A., Yau, C., et al. (2012) Lobular Histology and Response to Neoadjuvant Chemotherapy in Invasive Breast Cancer. Breast Cancer Research and Treatment, 136, 35-43. https://doi.org/10.1007/s10549-012-2233-Z

[19] Dixon, J.M., Renshaw, L., Dixon, J. and Thomas, J. (2011) Invasive Lobular Carcinoma: Response to Neoadjuvant Letrozole Therapy. Breast Cancer Research and Treatment, 130, 871-877. https://doi.org/10.1007/s10549-011-1735-4

[20] Cataliotti, L., Buzdar, A.U., Noguchi, S., et al. (2006) Comparison of Anastrozole versus Tamoxifen as Preoperative Therapy in Postmenopausal Women with Hormone Receptor-Positive Breast Cancer: The Pre-Operative "Arimidex" Compared to Tamoxifen (PROACT) Trial. Cancer, 106, 2095-2103.

https://doi.org/10.1002/cncr.21872

[21] National Comprehensive Cancer Network (2016) Clinical Practice Guidelines in Oncology: Breast Cancer (Version 2.2016).

https://www.nccn.org/professionals/physician gls/pdf/breast.pdf

[22] Deogracias, M., Jaime, L., Camisón, I., Zamacola, I., Murillo Guibert, J. and Suescun García, R. (2010) Rectal Metastasis from Lobular Breast Carcinoma 15 Years after Primary Diagnosis. Clinical and Translational Oncology, 12, 150-153. https://doi.org/10.1007/S12094-010-0481-0

[23] Taal, B.G., den Hartog Jager, F.C.A., Steinmetz, R. and Peterse, H. (1992) The Spectrum of Gastrointestinal Metastases of Breast Carcinoma. Gastrointestinal Endoscopy, 38, 136-141. https://doi.org/10.1016/S0016-5107(92)70378-2 